PROCEEDINGS OF THE

AMERICAN MATHEMATICAL SOCIETY

Volume 132, Number 9, Pages 2683-2691

S 0002-9939(04)07392-7

Article electronically published on April 9, 2004

\title{
THE LINEAR HEAT EQUATION WITH HIGHLY OSCILLATING POTENTIAL
}

ISMAIL KOMBE

(Communicated by Carmen C. Chicone)

Abstract. In this paper we consider the following initial value problem:

$$
\begin{cases}\frac{\partial u}{\partial t}=-H u+V(x) u & \text { in } \quad \mathbb{R}^{N} \times(0, T), \\ u(x, 0)=u_{0}(x) \geq 0 & \text { on } \quad \mathbb{R}^{N} \times\{t=0\},\end{cases}
$$

where $H=-\Delta-\frac{\beta}{|x|^{2}} \sin \left(\frac{1}{|x|^{\alpha}}\right)$ and $0 \leq V \in L_{\mathrm{loc}}^{1}\left(\mathbb{R}^{N}\right)$. Nonexistence of positive solutions is analyzed.

\section{INTRODUCTION}

Recently there has been great interest in the investigation of existence and nonexistence of positive solutions for the linear heat equation with a potential, e.g. [3], [], [9] and [6]. The motivating work for these papers is the classical paper of Baras and Goldstein [2], in which it was shown that the initial value problem

$$
\begin{cases}\frac{\partial u}{\partial t}=\Delta u+\frac{c}{|x|^{2}} u & \text { in } \quad \mathbb{R}^{N} \times(0, T), \\ u(x, 0)=u_{0}(x) \geq 0 \quad \text { in } \quad & \mathbb{R}^{N}\end{cases}
$$

has no nonnegative solutions except $u \equiv 0$ if $c>C^{*}(N)=\left(\frac{N-2}{2}\right)^{2}$. Moreover, all positive solutions blow up instantaneously, in the sense that if $u_{n}$ is the solution of the same problem with the potential $c /|x|^{2}$ replaced by $V_{n}=\min \left\{c /|x|^{2}, n\right\}$, then $\lim _{n \rightarrow \infty} u_{n}(x, t)=\infty$ for all $x \in \mathbb{R}^{N}$ and $t>0$. If $c \leq C^{*}(N)$, positive weak solutions do exist. Thus $C^{*}(N)=\left(\frac{N-2}{2}\right)^{2}$ is the cutoff point for existence of positive solutions for the heat equation with inverse square potential $c /|x|^{2}$, where $C^{*}(N)$ is also the sharp constant in Hardy's inequality. Note that by Hardy's inequality, the infimum of the expression

$$
\frac{\int_{\mathbb{R}^{N}}|\nabla \phi(x)|^{2} d x-\int_{\mathbb{R}^{N}} \frac{c}{|x|^{2}}|\phi(x)|^{2} d x}{\int_{\mathbb{R}^{N}}|\phi(x)|^{2} d x}
$$

over $0 \not \equiv \phi \in C_{c}^{\infty}\left(\mathbb{R}^{N} \backslash\{0\}\right)$ is either $-\infty$ or 0 , depending on whether $c>C^{*}(N)$ or $c \leq C^{*}(N)$.

Cabré and Martel [3] extended the above result to general positive singular potentials. They discovered that existence and nonexistence of positive solutions is

Received by the editors April 21, 2003 and, in revised form, June 18, 2003.

2000 Mathematics Subject Classification. Primary 35K15, 35K25, 35R25.

Key words and phrases. Heat equation, instantaneous blow up, positive solutions.

(C)2004 American Mathematical Society 
largely determined by the size of the infimum of the spectrum of the symmetric operator $S=-\Delta-V$, which is

$$
\sigma_{\mathrm{inf}}=\sigma_{\mathrm{inf}}(V ; \Omega)=\inf _{0 \neq \phi \in Q} \frac{\int_{\Omega}|\nabla \phi|^{2} d x-\int_{\Omega} V|\phi|^{2} d x}{\int_{\Omega}|\phi|^{2} d x} .
$$

Here $Q$ is a core of $S$. In dimension $N \geq 3$, one has $Q=C_{0}^{\infty}(\Omega)$; however, for $0 \in \Omega \subset \mathbb{R}^{N}$ and $N \leq 2$, it is convenient to take $Q=C_{0}^{\infty}(\Omega \backslash\{0\})$.

Goldstein and Zhang [8] have found an analogue of the results for (1.1) on the Heisenberg group. Namely,

$$
\left\{\begin{array}{l}
u_{t}(z, l, t)=\Delta_{\mathbb{H}^{n}} u(z, l, t)-\frac{c|z|^{2}}{|z|^{4}+l^{2}} u(z, l, t), \\
u(z, l, 0)=u_{0}(z, l), \quad(z, l)=(x, y, l) \in \mathbb{H}^{n}, \quad t \in(0, T], \quad T>0,
\end{array}\right.
$$

has no positive solution if $c>C_{*}(n)=n^{2}$. Moreover, they have shown that all positive solutions blow up instantaneously, and if $c \leq C_{*}(n)$, then positive weak solutions do exist.

Recently, Goldstein and Zhang 9] have extended the method of Cabré and Martel [3] to uniformly parabolic equations with positive singular potentials. Our work in this paper has been influenced by [2], [3], [8], and [9] with the following question:

- Is it possible to perturb the positive potential by a singular and sign changing potential that does not effect the nonexistence of positive solutions?

We answer this question in this paper.

In the same context there are also some related works on nonlinear parabolic problems by Garcia and Peral [5, Aguilar and Peral [1, Goldstein and Kombe [7.

\section{Highly Singular, oscillating POtentials}

In the present paper we consider the initial value problem

$$
\begin{cases}\frac{\partial u}{\partial t}=-H u+V(x) u & \text { in } \quad \mathbb{R}^{N} \times(0, T), \\ u(x, 0)=u_{0}(x) \geq 0 & \text { on } \quad \mathbb{R}^{N} \times\{t=0\},\end{cases}
$$

where $N \geq 3, u_{0}(x)$ is not identically zero, and $0 \leq V(x) \in L_{\text {loc }}^{1}\left(\mathbb{R}^{N}\right)$. The operator $H$ is the symmetric operator (defined on $C_{c}^{\infty}\left(\mathbb{R}^{N} \backslash\{0\}\right)$ )

$$
H=-\Delta-\frac{\beta}{|x|^{2}} \sin \left(\frac{1}{|x|^{\alpha}}\right),
$$

where $\alpha>0$ and $\beta \in \mathbb{R} \backslash\{0\}$. Although the potential $V_{\text {osc }}=\frac{\beta}{|x|^{2}} \sin \left(\frac{1}{|x|^{\alpha}}\right)$ is highly singular and oscillates wildly, a remarkable result has been obtained by K.-T. Sturm [12]. He proved that $H$ satisfies the Gaussian bounds

$$
\gamma_{1} e^{\alpha_{1} t} G_{0}\left(x, y, \beta_{1} t\right) \leq G_{\mathrm{osc}}\left(x, y, t ; V_{\mathrm{osc}}\right) \leq \gamma_{2} e^{\alpha_{2} t} G_{0}\left(x, y, \beta_{2} t\right)
$$

for all $(x, y, t) \in \mathbb{R}^{N} \times \mathbb{R}^{N} \times(0, \infty)$, where $\beta_{1}, \beta_{2}, \gamma_{1}, \gamma_{2} \in \mathbb{R}_{+} \backslash\{0\} ; \alpha_{1}, \alpha_{2} \in \mathbb{R}$; $G_{\text {osc }}$ is the Green's function for $H$, and

$$
G_{0}(x, y, t)=(4 \pi t)^{-N / 2} \exp \left(-|x-y|^{2} /(4 t)\right)
$$

is the Green's function for $-\Delta$ on $\mathbb{R}^{N}$.

Before proceeding to the main results of the paper, we present the definition of a weak solution. 
Definition 2.1. We say that $u(\cdot, t)$ is a weak solution to $(2.1)$ if

$$
u,|\nabla u| \in L_{\mathrm{loc}}^{2}\left(\mathbb{R}^{N} \times(0, T)\right), \quad V(\cdot) u(\cdot, t) \in L_{\mathrm{loc}}^{1}\left(\mathbb{R}^{N} \times(0, T)\right)
$$

and

$$
\begin{aligned}
& \int_{t_{1}}^{t_{2}} \int_{\mathbb{R}^{N}} u \phi_{t} d x d t-\left.\int_{\mathbb{R}^{N}}(u \phi)\right|_{t_{1}} ^{t_{2}} d x+\int_{t_{1}}^{t_{2}} \int_{\mathbb{R}^{N}} \nabla u \cdot \nabla \phi d x d t \\
& =\int_{t_{1}}^{t_{2}} \int_{\mathbb{R}^{N}}\left(V+\frac{\beta}{|x|^{2}} \sin \left(\frac{1}{|x|^{\alpha}}\right)\right) u \phi d x d t
\end{aligned}
$$

for all compactly supported $C^{1,1}$-functions $\phi$ and $0<t_{1}<t_{2}<T$.

In general we approach the problem in (2.1) via the approximate problem

$$
\begin{cases}\frac{\partial u_{k}}{\partial t}=-H u_{k}+V_{k}(x) u_{k} & \text { in } \mathbb{R}^{N} \times(0, \infty), \\ u_{k}(x, 0)=u_{0}(x) & \text { on } \quad \mathbb{R}^{N} \times\{t=0\},\end{cases}
$$

where $V_{k}$ is bounded above and $V_{k}(x) \uparrow V(x)$ for all $x \in \mathbb{R}^{N}$. Maximum principle arguments show that $u_{k}$ exists (if $u_{0}$ is not too singular), is the unique positive solution to $(2.4)$, and $u_{k}(x, t) \leq u(x, t)$ for all $(x, t) \in \mathbb{R}^{N} \times[0, \infty)$ if $u(x, t)$ exists; and the sequence $u_{k}(x, t)$ is nondecreasing in $k$.

Definition 2.2. We say that (2.1) has instantaneous blow-up if $\lim _{k \rightarrow \infty} u_{k}(x, t)=$ $\infty$ for all $(x, t) \in \mathbb{R}^{N} \times(0, \infty)$.

The following is our main result.

Theorem 2.3. Let $N \geq 3,0 \leq V \in L_{\text {loc }}^{1}\left(\mathbb{R}^{N} \backslash \mathcal{K}\right)$, and $Q=\left\{\phi \in C_{c}^{\infty}\left(\mathbb{R}^{N} \backslash \mathcal{K}\right)\right.$ : $\phi$ real, $\phi \not \equiv 0\}$, where $\mathcal{K}$ is a finite set. If

$$
\sigma_{\inf }\left(V ; \mathbb{R}^{N} \backslash \mathcal{K}\right):=\inf _{\phi \in Q} \frac{\int_{\mathbb{R}^{N}}\left(|\nabla \phi|^{2}-\left(1-\epsilon_{1}\right) V|\phi|^{2}\right) d x}{\int_{\mathbb{R}^{N}}|\phi|^{2} d x}=-\infty
$$

for some $\epsilon_{1}>0$, then the problem (2.1) has no positive solutions. Moreover, we have instantaneous blow-up.

\section{Proof of Theorem 2.3}

The proof is by contradiction. Given any $T>0$, let $u$ be a solution to (2.1) in $\mathbb{R}^{N} \times(0, T)$ with $u_{0} \geq 0$ but not identically zero. First we will proceed by approximation. We truncate $V$ as

$$
V_{k}=\min \{V, k\}, \quad k \in \mathbb{N},
$$

and let $\left\{u_{k}\right\}_{k \in \mathbb{N}}$ be the unique positive solution of (2.4). We must show that $u_{k}(x, t) \longrightarrow \infty$ for all $x \in \mathbb{R}^{N}$ and $t>0$.

Let us multiply both sides of (2.4) by the test function $\phi^{2} / u_{k}$ with $\phi \in Q$, and integrate over $\mathbb{R}^{N}$ :

$$
\int_{\mathbb{R}^{N}}\left(V_{k}(x)+V_{\mathrm{osc}}(x)\right) \phi^{2}(x) d x=\partial_{t} \int_{\mathbb{R}^{N}}\left(\log u_{k}\right) \phi^{2} d x-\int_{\mathbb{R}^{N}} \Delta u_{k}\left(\frac{\phi^{2}}{u_{k}}\right) d x .
$$

Using integration by parts, we get

$$
\int_{\mathbb{R}^{N}} \Delta u_{k}\left(\frac{\phi^{2}}{u_{k}}\right) d x=-\int_{\mathbb{R}^{N}} \nabla u_{k} \cdot \nabla\left(\frac{\phi^{2}}{u_{k}}\right) d x .
$$


Indeed, a direct computation shows that

$$
\begin{aligned}
\int_{\mathbb{R}^{N}} \Delta u_{k}\left(\frac{\phi^{2}}{u_{k}}\right) d x & =\int_{\mathbb{R}^{N}}\left|\nabla u_{k}\right|^{2} \frac{\phi^{2}}{u_{k}^{2}} d x-2 \int_{\mathbb{R}^{N}}\left(\nabla u_{k} \cdot \nabla \phi\right) \frac{\phi}{u_{k}} d x \\
& \geq-\int_{\mathbb{R}^{N}}|\nabla \phi(x)|^{2} d x .
\end{aligned}
$$

Substituting (3.2) into (3.1), we obtain

$$
\int_{\mathbb{R}^{N}} V_{k}(x) \phi^{2}(x) d x+\int_{\mathbb{R}^{N}} V_{\text {osc }}(x) \phi^{2}(x) d x-\int_{\mathbb{R}^{N}}|\nabla \phi(x)|^{2} d x \leq \partial_{t} \int_{\mathbb{R}^{N}}\left(\log u_{k}\right) \phi^{2} d x .
$$

Integrating from $t_{1}$ to $t_{2}\left(0<t_{1}<t_{2}\right)$, we get

$$
\begin{aligned}
& \int_{\mathbb{R}^{N}} V_{k}(x) \phi^{2} d x+\int_{\mathbb{R}^{N}} V_{\mathrm{osc}}(x) \phi^{2}(x) d x-\int_{\mathbb{R}^{N}}|\nabla \phi(x)|^{2} d x \\
& \leq \frac{1}{t_{2}-t_{1}} \int_{\mathbb{R}^{N}} \log \left(\frac{u_{k}\left(x, t_{2}\right)}{u_{k}\left(x, t_{1}\right)}\right) \phi^{2}(x) d x .
\end{aligned}
$$

From here on, we will use $L_{w}^{1}\left(\mathbb{R}^{N}\right)=\left\{f \in \mathbb{R}^{N}: \int_{\mathbb{R}^{N}}|f(x)| e^{-|x|} d x<\infty\right\}$, the $L^{1}$ space with weight $w(x) \equiv e^{-|x|}$. Let $\rho \in C_{0}\left(\mathbb{R}^{N}\right)$ be any strictly positive function in $\mathbb{R}^{N}$ such that $\rho$ converges to zero at $\infty$ and $\log \rho \in L_{w}^{p}\left(\mathbb{R}^{N}\right)$ for any $p>1$.

Let $T>0$. We claim that there exists at most one point $t_{1} \in(0, T)$ such that $u\left(\cdot, t_{1}\right) \rho(\cdot) \in L_{w}^{1}\left(\mathbb{R}^{N}\right)$. Suppose the contrary. Then there exist $t_{1}, t_{2} \in(0, T)$ with $t_{2}>t_{1}$ such that

$$
u\left(\cdot, t_{i}\right) \rho(\cdot) \in L_{w}^{1}\left(\mathbb{R}^{N}\right), \quad i=1,2 .
$$

Next, we will prove that

$$
\log \left(u_{k}\left(\cdot, t_{i}\right) \rho(\cdot)\right) \in L_{w}^{p}\left(\mathbb{R}^{N}\right), \quad p \geq 1 .
$$

Let us write

$$
\mathbb{R}^{N}=S_{1} \cup S_{2} \equiv\left\{x \in \mathbb{R}^{N} \mid u_{k}\left(x, t_{1}\right) \rho(x) \geq 1\right\} \cup\left\{x \in \mathbb{R}^{N} \mid u_{k}\left(x, t_{1}\right) \rho(x)<1\right\} .
$$

Using Jensen's inequality for the concave function $\log ^{p}(s)$ over the set $S_{1}$, we get

$$
\int_{S_{1}}\left|\log \left(u_{k}\left(x, t_{i}\right) \rho(x)\right)\right|^{p} w(x) d x \leq C \log ^{p}\left(\int_{S_{1}}\left|u_{k}\left(x, t_{i}\right) \rho(x)\right| w(x) d x\right)<\infty,
$$

where $C$ is independent of $k$ and we have used the fact that $S_{1}$ has finite Lebesgue measure.

When $x \in S_{2}$, we can write

$$
1>u_{k}\left(x, t_{1}\right) \rho(x)=\int_{\mathbb{R}^{N}} G\left(x, y, t_{1} ; W_{k}\right) u_{0}(y) \rho(x) d y,
$$

where $G$ is the Green's function of (2.4) and $W_{k}=V_{k}+V_{\text {osc }}$. By the maximum principle,

$$
\int_{\mathbb{R}^{N}} G\left(x, y, t_{1} ; W_{k}\right) u_{0}(y) \rho(x) d y \geq \int_{\mathbb{R}^{N}} G_{\mathrm{osc}}\left(x, y, t_{1} ; V_{\mathrm{osc}}\right) u_{0}(y) \rho(x) d y .
$$

Using the Gaussian estimates (2.3), we obtain

$$
\int_{\mathbb{R}^{N}} G\left(x, y, t_{1} ; V_{\mathrm{osc}}\right) u_{0}(y) \rho(x) d y \geq \gamma e^{\lambda t_{1}} \int_{\mathbb{R}^{N}} G_{0}\left(x, y, \beta t_{1}\right) u_{0}(y) \rho(x) d y,
$$

where $G_{0}$ is the heat kernel of $u_{t}-\Delta u=0$ and $\gamma>0, \beta>0, \lambda \in \mathbb{R}$. 
Let us define $h$ by

$$
h\left(x, \beta t_{1}\right) \rho(x) \equiv \int_{\mathbb{R}^{N}} G_{0}\left(x, y, \beta t_{1}\right) u_{0}(y) \rho(x) d y .
$$

Then we have

$$
\left|\log \left(u_{k}\left(x, t_{1}\right) \rho(x)\right)\right| \leq|\log (\gamma)|+|\lambda| t_{1}+\left|\log \left(h\left(x, \beta t_{1}\right) \rho(x)\right)\right| .
$$

Clearly, $\log (\gamma)$ and $\lambda t_{1} \in L_{w}^{p}\left(\mathbb{R}^{N}\right)$. We need to show that

$$
\log h\left(x, \beta t_{1}\right) \in L_{w}^{p}\left(S_{2}\right)
$$

for any $p>1$.

If we assume $u_{0} \leq c$, then $h(x, t) \leq c_{2}(t)$ follows immediately. But we want to minimize the number of assumptions. According to the strong maximum principle, there is, for any $t>0$, a positive constant $c_{1}(t)$ such that

$$
h(0, t / 2) \geq c_{1}(t) .
$$

We connect $\left(0, \frac{t}{2}\right)$ and $(x, t)$ by a line $l$. Consider the points on $l$

$$
x_{j}=\frac{2 j x}{k|x|}, \quad t_{j}=\frac{t}{2}+\frac{j t}{k|x|}, \quad j=0,1, \ldots, J ; \quad J-1<\frac{k|x|}{2} \leq J \in \mathbb{N},
$$

where $k>0$ is a suitable number. We can use the following Harnack inequality (see [11] or [10]):

$$
\log \frac{h\left(x_{j}, t_{j}\right)}{h\left(x_{j+1}, t_{j+1}\right)} \leq c\left[\frac{\left|x_{j+1}-x_{j}\right|^{2}}{t_{j+1}-t_{j}}+\log \left(\frac{t_{j+1}}{t_{j}}\right)+1\right],
$$

where $0<t_{j}<t_{j+1}<T$ and $c>0$. Clearly we can write

$$
h\left(x_{j}, t_{j}\right) \leq h\left(x_{j+1}, t_{j+1}\right) \exp \left[c\left(\frac{\left|x_{j+1}-x_{j}\right|^{2}}{t_{j+1}-t_{j}}+\frac{t_{j+1}}{t_{j}}+1\right)\right], \quad j=0, \ldots, J .
$$

Iterating $J$ times, we obtain

$$
\begin{gathered}
h\left(x_{0}, t_{0}\right) \leq h\left(x_{1}, t_{1}\right) \exp \left[c\left(\frac{\left|x_{1}-x_{0}\right|^{2}}{t_{1}-t_{0}}+\frac{t_{1}}{t_{0}}+1\right)\right] \\
\vdots \\
c_{1}(t) \leq h\left(x_{0}, t_{0}\right) \leq h(x, t) \exp \left(\frac{2 c|x|^{2}}{t}\right) \exp (k c|x|) .
\end{gathered}
$$

Note that

$$
\left|x_{j+1}-x_{j}\right|=\frac{2}{k}, \quad\left|t_{j+1}-t_{j}\right|=\frac{t}{k|x|}, \quad j=0, \ldots, J
$$

and

$$
\begin{aligned}
\exp \left(c \sum \frac{t_{j+1}}{t_{j}}\right) & \leq \exp \left(\frac{c}{t_{0}} \sum t_{j+1}\right), \quad j=0, \ldots, J, \\
& \leq \exp (c k|x|) .
\end{aligned}
$$

We can choose $k=1 / c$, and therefore

$$
h(x, t) \geq c_{1}(t) \exp (-|x|) \exp \left(-2 c \frac{|x|^{2}}{t}\right) .
$$

This is a lower bound. Next we find an upper bound for $h(x, t)$ using the same technique. By the above,

$$
h(0,2 t) \geq c_{2}(t)
$$


We connect $(0,2 t)$ and $(x, t)$ by a line $l$. Consider the points on $l$ where $x \neq 0$ :

$$
x_{j}=x\left(1-\frac{j}{k|x|}\right), \quad t_{j}=t+\frac{j t}{k|x|}, \quad j=0,1, \ldots, J ; \quad J-1<k|x| \leq J \in \mathbb{N},
$$

where $k>0$ is a suitable number. Iterating (3.8), we get

$$
h(x, t) \leq c_{2}(t) \exp (2 k|x|) \exp \left(c \frac{|x|^{2}}{t}\right),
$$

and choosing $k=1 / c$ we obtain

$$
c_{1}(t) \exp (-|x|) \exp \left(-2 c \frac{|x|^{2}}{t}\right) \leq h(x, t) \leq c_{2}(t) \exp (2|x|) \exp \left(c \frac{|x|^{2}}{t}\right) .
$$

Clearly

$$
|\log h(x, t)| \leq c_{1}(t)+|x|+2 c \frac{|x|^{2}}{t} .
$$

Hence

$$
\int_{S_{2}}|\log h(x, t)|^{p} w(x) d x \leq C .
$$

This proves that

$$
\int_{S_{2}}\left|\log \left(u_{k}\left(x, t_{1}\right) \rho(x)\right)\right|^{p} w(x) d x \leq C .
$$

Repeating this process for $u_{k}\left(\cdot, t_{2}\right)$, we conclude that

$$
\log \frac{u_{k}\left(\cdot, t_{2}\right)}{u_{k}\left(\cdot, t_{1}\right)}=\log \left(u_{k}\left(\cdot, t_{2}\right) \rho(\cdot)\right)-\log \left(u_{k}\left(\cdot, t_{1}\right) \rho(\cdot)\right) \in L_{w}^{p}\left(\mathbb{R}^{N}\right)
$$

for any $p>1$.

Let us write

$$
\varphi \equiv w(x) \frac{1}{t_{2}-t_{1}}\left(\log \frac{u_{k}\left(x, t_{2}\right)}{u_{k}\left(x, t_{1}\right)}\right) .
$$

We know that $\varphi \in L^{p}\left(\mathbb{R}^{N}\right)$ for any $p>1$. We will show that, for any $\epsilon>0$, there exists $C(\epsilon)>0$ such that

$$
\int_{\mathbb{R}^{N}} \varphi \phi^{2} d x \leq \frac{\epsilon}{(1-\epsilon)} \int_{\mathbb{R}^{N}}|\nabla \phi(x)|^{2} d x+C(\epsilon) \int_{\mathbb{R}^{N}} \phi^{2}(x) d x
$$

for any $\phi \in W_{0}^{1,2}\left(\mathbb{R}^{N}\right)$.

Here is the proof. Given $\epsilon>0$, we choose $R$ sufficiently large so that

$$
\int_{\mathbb{R}^{N} \backslash B\left(x_{0}, R\right)} \varphi^{\frac{N}{2}}(x) d x \leq\left(\frac{\epsilon}{2 c_{N}(1-\epsilon)}\right)^{\frac{N}{2}},
$$

where $c_{N}$ is an appropriate constant which will be chosen later. We now use the following a priori inequality, which is an easy consequence of the Sobolev embedding theorem (see [9]). For every $\epsilon>0$, there exists $C(\epsilon)$ such that

$$
\begin{aligned}
& \int_{B\left(x_{0}, R\right)} \varphi(x) \phi^{2}(x) d x \\
& \leq \frac{\epsilon}{2(1-\epsilon)} \int_{B\left(x_{0}, R\right)}|\nabla \phi(x)|^{2} d x+C(\epsilon) \int_{B\left(x_{0}, R\right)} \phi^{2}(x) d x .
\end{aligned}
$$


We write

$$
\int_{\mathbb{R}^{N}} \varphi \phi^{2} d x=\int_{\mathbb{R}^{N} \backslash B\left(x_{0}, R\right)} \varphi(x) \phi^{2}(x) d x+\int_{B\left(x_{0}, R\right)} \varphi(x) \phi^{2}(x) d x .
$$

Applying Hölder's inequality to

$$
\int_{\mathbb{R}^{N} \backslash B\left(x_{0}, R\right)} \varphi(x) \phi^{2}(x) d x,
$$

we obtain

$$
\int_{\mathbb{R}^{N} \backslash B\left(x_{0}, R\right)} \varphi(x) \phi^{2}(x) d x \leq\left(\int_{\mathbb{R}^{N} \backslash B\left(x_{0}, R\right)} \varphi^{\frac{N}{2}}(x) d x\right)^{\frac{2}{N}}\left(\int_{\mathbb{R}^{N}} \phi^{\frac{2 N}{N-2}}(x) d x\right)^{\frac{N-2}{N}} .
$$

Applying the Gagliardo-Nirenberg-Sobolev inequality 44 leads to

$$
\left(\int_{\mathbb{R}^{N}} \phi(x)^{\frac{2 N}{N-2}}(x) d x\right)^{\frac{N-2}{N}} \leq c_{N} \int_{\mathbb{R}^{N}}|\nabla \phi|^{2} d x
$$

for some constant $c_{N}$. We now recall (3.11); this is the $c_{N}$ appearing there.

Therefore,

$$
\int_{\mathbb{R}^{N} \backslash B\left(x_{0}, R\right)} \varphi(x) \phi^{2}(x) d x \leq \frac{\epsilon}{2(1-\epsilon)} \int_{\mathbb{R}^{N}}|\nabla \phi(x)|^{2} d x .
$$

Substituting (3.12) and (3.14) into (3.13) gives the desired inequality (3.10). Substituting (3.10) into (3.3) and passing to the limit, we obtain

$$
\begin{aligned}
& \int_{\mathbb{R}^{N}} V(x) \phi^{2}(x) d x+\int_{\mathbb{R}^{N}} V_{\mathrm{osc}}(x) \phi^{2}(x) d x-\int_{\mathbb{R}^{N}}|\nabla \phi(x)|^{2} d x \\
& \leq \frac{\epsilon}{1-\epsilon} \int_{\mathbb{R}^{N}}|\nabla \phi(x)|^{2} d x+C(\epsilon) \int_{\mathbb{R}^{N}} \phi^{2}(x) d x .
\end{aligned}
$$

Clearly

$$
\begin{aligned}
& \frac{1}{1-\epsilon} \int_{\mathbb{R}^{N}}|\nabla \phi(x)|^{2} d x-\int_{\mathbb{R}^{N}} V(x) \phi^{2}(x) d x-\int_{\mathbb{R}^{N}} V_{\text {osc }}(x) \phi^{2}(x) d x \\
& \geq-C(\epsilon) \int_{\mathbb{R}^{N}} \phi^{2}(x) d x .
\end{aligned}
$$

We can rearrange (by letting $(1-\epsilon) \beta=\epsilon \tilde{\beta})$ :

$$
\begin{aligned}
& (1+\epsilon) \int_{\mathbb{R}^{N}}|\nabla \phi|^{2} d x-\int_{\mathbb{R}^{N}}(1-\epsilon) V \phi^{2} d x-\epsilon\left(\int_{\mathbb{R}^{N}}|\nabla \phi|^{2} d x-\int_{\mathbb{R}^{N}} \tilde{V}_{\mathrm{osc}} \phi^{2} d x\right) \\
& \geq-C(\epsilon)(1-\epsilon) \int_{\mathbb{R}^{N}} \phi^{2} d x,
\end{aligned}
$$

where

We write

$$
\tilde{V}_{\mathrm{osc}}=\left(\frac{1-\epsilon}{\epsilon}\right) V_{\mathrm{osc}}(x)=\frac{\tilde{\beta}}{|x|^{2}} \sin \left(\frac{1}{|x|^{\alpha}}\right) .
$$

$$
\begin{aligned}
& \int_{\mathbb{R}^{N}}|\nabla \phi|^{2} d x-\int_{\mathbb{R}^{N}}\left(\frac{1-\epsilon}{1+\epsilon}\right) V \phi^{2} d x \\
& \geq \frac{\epsilon}{1+\epsilon}\left(\int_{\mathbb{R}^{N}}|\nabla \phi|^{2} d x-\int_{\mathbb{R}^{N}} V_{\mathrm{osc}} \phi^{2} d x\right)-\frac{C(\epsilon)(1-\epsilon)}{1+\epsilon} \int_{\mathbb{R}^{N}} \phi^{2} d x .
\end{aligned}
$$


The quadratic form for $H$ is bounded from below [12], i.e.,

$$
\int_{\mathbb{R}^{N}}|\nabla \phi|^{2} d x-\int_{\mathbb{R}^{N}} V_{\mathrm{osc}} \phi^{2} d x \geq l \int_{\mathbb{R}^{N}} \phi^{2} d x
$$

where $l \in \mathbb{R}$. Therefore, for some $\epsilon_{1}>0$, we have

$$
\frac{\int_{\mathbb{R}^{N}}|\nabla \phi|^{2} d x-\int_{\mathbb{R}^{N}}\left(1-\epsilon_{1}\right) V \phi^{2} d x}{\int_{\mathbb{R}^{N}} \phi^{2} d x} \geq-C_{1}\left(\epsilon, \epsilon_{1}\right)>-\infty .
$$

This contradicts our assumption in Theorem 2.3. The claim is proven, i.e., there is at most one $t_{1}>0$ such that $u\left(\cdot, t_{1}\right) \rho(\cdot) \in L_{w}^{1}\left(\mathbb{R}^{N}\right)$.

Now we will show that $u_{k}(x, t) \longrightarrow \infty$ for all $(x, t) \in \mathbb{R}^{N} \times(0, \infty)$.

Given $x \in \mathbb{R}^{N}$ and $t \in(0, T)$, we have

$$
u_{k}(x, t)=\int_{\mathbb{R}^{N}} G\left(x, y, t ; V_{k}\right) u_{k}(y, t) w(y) d y,
$$

where $G\left(x, y, t ; V_{k}\right)$ is the heat kernel of $\frac{\partial u_{k}}{\partial t}-\Delta u_{k}=V_{k} u_{k}$. Using the maximum principle and the Gaussian bounds (2.3), we see that

$$
u_{k}(x, t) \geq \gamma e^{\lambda t} \int_{\mathbb{R}^{N}} G_{0}\left(x, y, \frac{\beta t}{2}\right) u_{k}\left(y, \frac{t}{2}\right) \rho(y) w(y) d y
$$

where $\gamma>0, \beta>0, \lambda \in \mathbb{R}$ and $G_{0}$ is the heat kernel of $\Delta u-u_{t}=0$. Since $u(x, t)$ is the monotone limit of the $u_{k}(x, t)$, we can write

$$
u(x, t) \geq \gamma e^{\lambda t} \int_{\mathbb{R}^{N}} G_{0}\left(x, y, \frac{\beta t}{2}\right) u\left(y, \frac{t}{2}\right) \rho(y) d y .
$$

We take $\rho=\rho(y)=G_{0}\left(x, y, \frac{\beta t}{2}\right)$. By the strong maximum principle, $\rho(y)>0$ for any $y \in \mathbb{R}^{N}$.

If there is no $s \in\left(0, \frac{t}{2}\right]$ such that $\rho(\cdot) u(\cdot, s) w(\cdot) \in L^{1}\left(\mathbb{R}^{N}\right)$, then

$$
u(x, t) \geq \gamma e^{\lambda t} \int_{\mathbb{R}^{N}} G_{0}\left(x, y, \frac{\beta t}{2}\right) u\left(y, \frac{t}{2}\right) w(y) d y=\gamma e^{\lambda t} \int_{\mathbb{R}^{N}} \rho(y) u\left(y, \frac{t}{2}\right) w(y) d y=\infty .
$$

If $s \in\left(0, \frac{t}{2}\right]$ is the only point such that $\rho(\cdot) u(\cdot, s) w(y) \in L^{1}\left(\mathbb{R}^{N}\right)$, then

$$
\begin{aligned}
u\left(x, \frac{t+s}{2}\right) & \geq \gamma e^{\lambda t} \int_{\mathbb{R}^{N}} G_{0}\left(x, y, \frac{\beta t}{2}\right) u\left(y, \frac{s}{2}\right) w(y) d y \\
& =\gamma e^{\lambda t} \int_{\mathbb{R}^{N}} \rho(y) u\left(y, \frac{s}{2}\right) w(y) d y \\
& =\infty .
\end{aligned}
$$

By the Harnack inequality [10, for any small $\delta>0$, there exist $c, r>0$ such that

$$
G_{0}\left(z, y, \frac{\beta(t+\delta)}{2}\right) \geq c G_{0}\left(x, y, \frac{\beta t}{2}\right)
$$

when $z \in B(x, r) \subset \mathbb{R}^{N}$. Therefore, for $z \in B(x, r) \subset \mathbb{R}^{N}$,

$$
\begin{aligned}
u\left(z, \frac{t+s+\delta}{2}\right) & \geq \gamma e^{\lambda t} \int_{\mathbb{R}^{N}} G_{0}\left(z, y, \frac{\beta(t+\delta)}{2}\right) u\left(y, \frac{s}{2}\right) w(y) d y \\
& \geq c \gamma e^{\lambda t} \int_{\mathbb{R}^{N}} G_{0}\left(x, y, \frac{\beta t}{2}\right) u\left(y, \frac{s}{2}\right) w(y) d y=\infty
\end{aligned}
$$


By the reproducing formula again, we know that

$$
\begin{aligned}
u(x, t) & \geq \int_{\mathbb{R}^{N}} G_{0}\left(x, z, \frac{t-s-\delta}{2}\right) u\left(z, \frac{t+s+\delta}{2}\right) w(z) d z \\
& \geq \int_{|x-z| \leq r} G_{0}\left(x, z, \frac{t-s-\delta}{2}\right) u\left(z, \frac{t+s+\delta}{2}\right) w(z) d z=\infty .
\end{aligned}
$$

Since $(x, t)$ is arbitrary, this proves the blow-up part.

We now have the following corollary as an immediate consequence of Theorem 2.3 .

Corollary 3.1. Let $0 \in \Omega, N \geq 3, C^{*}(N)=\left(\frac{N-2}{2}\right)^{2}$, and $V(x)=\frac{c}{|x|^{2}}$. Then the problem (2.1) has no positive solutions if $c>C^{*}(N)$.

Remark 3.1. In the paper [6], Goldstein and Kombe conjectured that if $c$ decreases to below $C^{*}(N)$ and $|\beta|$ is large enough, then there is instantaneous blow-up. We now see that in the light of Theorem 2.3, this is not so. Namely, we cannot decrease $c$ to $c<C^{*}(N)$ no matter how large we choose $\beta$ to get instantaneous blow-up.

\section{ACKNOWLEDGEMENT}

I am indebted to my thesis advisor Jerome A. Goldstein for helpful discussions leading to this article and for his constant support. Furthermore, I would like to thank Qi S. Zhang for helpful discussions.

\section{REFERENCES}

1. J. A. Aguilar Crespo and I. Peral Alonso, Global behaviour of the Cauchy problem for some critical nonlinear parabolic equations, SIAM J. Math. Anal., 31 (2000), 1270-1294. MR 2001d:35086

2. P. Baras and J. A. Goldstein, The heat equation with a singular potential, Trans. Amer. Math. Soc. 284 (1984), 121-139. MR 85f:35099

3. X. Cabré and Y. Martel, Existence versus explosion instantanée pour des équations de la chaleur linéaires avec potentiel singulier, C. R. Acad. Sci. Paris Sér. I. Math., 329 (1999), 973-978. MR 2000j:35117

4. L. C. Evans, Partial Differential Equations, Graduate Studies in Mathematics, vol. 19, American Mathematical Society, Providence, Rhode Island, 1998. MR 99e:35001

5. J. Garcia Azorero and I. Peral Alonso, Hardy inequalities and some critical elliptic and parabolic problems, J. Differential Equations, 144 (1998), 441-476. MR 99f:35099

6. J. A. Goldstein and Ismail Kombe, Instantaneous blow up, Advances in differential equations and mathematical physics (Birmingham, AL, 2002), Contemp. Math., Vol. 327, Amer. Math. Soc., Providence, RI, 2003, pp. 141-150.

7. J. A. Goldstein and I. Kombe, Nonlinear degenerate parabolic equations with singular lowerorder term, Advances in Differential Equations 8 (2003), 1153-1192.

8. J. A. Goldstein and Q. S. Zhang, On a degenerate heat equation with a singular potential, J. Functional Analysis, 186 (2001), 342-359. MR 2002k:35179

9. J. A. Goldstein and Q. S. Zhang, Linear parabolic equations with strong singular potentials, Trans. Amer. Math. Soc. 355 (2003), 197-211. MR 2003h:35096

10. G. M. Lieberman, Second Order Parabolic Differential Equations, World Scientific Publishing Co., Inc., River Edge, NJ, 1996. MR 98k:35003

11. J. Moser, A Harnack inequality for parabolic differential equations, Comm. Pure Appl. Math. 17 (1964), 101-134. MR 28:2357

12. K.-T. Sturm, Schrödinger operators with highly singular, oscillating potentials, Manuscripta Math. 76 (1992), 367-395. MR 94m:35077

Department of Mathematics, 202 Mathematical Sciences Building, University of MisSouri, Columbia, Missouri 65211

E-mail address: kombe@math.missouri.edu 